\title{
The Study of Product Availability in a Dual-channel Distribution Supply Chain with $(Q, R)$ Policy of Retailer
}

\author{
Fan He-hua and Zhou Yong-wei \\ Department of Mathematics and Physics, Zhengzhou Institute of Aeronautical \\ Industry Management, Zhengzhou, China 450015 \\ zwwfhh@126.com
}

\begin{abstract}
The paper focus on the supply chain model with a single product in electronic and retail channel, only a supplier and a retailer in system, customers have alternatives to choose the channel that is better suited to their needs, some of them may alternatively switch to the other channel when a stock-out occurs in their preferred channel. Supplier and retailer implement one-for-one and $(Q, R)$ ordering policies respectively to replenish their inventories. We set up the profit model of supply chain by Markov process, and study the product availability of supply chain. The result shows that the stock-out based substitution rate may increase or decrease the efficiency of decentralized supply chain; the centralized supply chain can adjust the based-stock levels timely to different stock-out based substitution rate and benefit from it, the supplier and retailer in a decentralized supply chain are more inattentive to customers' stock-out based substitution rate, and prone to lose their benefits.
\end{abstract}

Keywords: Stock-out based substitution; $(Q, R)$ policy; dual-channel; supply chain; basestock level

\section{Introduction}

With the advent of e-commerce and the rapid development of third-party logistics, more and more brand suppliers have began to set up direct sales by introducing internet channel. Such as IBM, HP, Nike, Dell and so on. Supplier sell products both through traditional retail and internet channel to customers, customers may choose the channel that is better suited to their needs, some of them may switch to another channel to buy product when a stock-out occurs in their favorite channel. Such a distribution model is called dual-channel supply chain distribution. It can increase the share of market, and know the more demand information for supplier, and can improve the brand competitiveness of product for sale.

In the stock-out based substitution dual-channel supply chain, there exist vertical and horizontal competition simultaneously between supplier and retailer (see Figure 1). Specifically, vertical competition mainly relates to the price double marginalization [1]. And horizontal competition mainly comes from that supplier and retailer sell the same product, and exists the competition in product inventory [2-4].

Anupindi and Ryzin studied the situation of vertical and horizontal competition coexist in a dual-channel supply chain [5-6], the result showed that when the two competition coexist, the inefficient understocking because of vertical competition can be counteracted by the overstocking due to horizontal competition in a supply chain, and help to improve the supply chain's performance. But the above researches are all assumed that supplier have no inventories, so the study results have some boundedness; Chiang W.K. and Kumar mainly studied the pricing policy and service coordination of dual-channel supply chain [7-8]; Kevin 
Chiang studied the inventory policy of dual-channel supply chain [9], but the author supposed that both supplier and retailer implement one-for-one ordering policies to replenish their inventories. The paper improves the stock-out based substitution supply chain model, in view of ordering cost, retailer implements $(\mathrm{Q}, \mathrm{R})$ ordering policy to replenish inventory. And studies the product availability of dual-channel supply based on the $(\mathrm{Q}, \mathrm{R})$ policy of retailer and stock-out based substitution, and hopes to provide theoretical guidance for the decision making of relative companies.

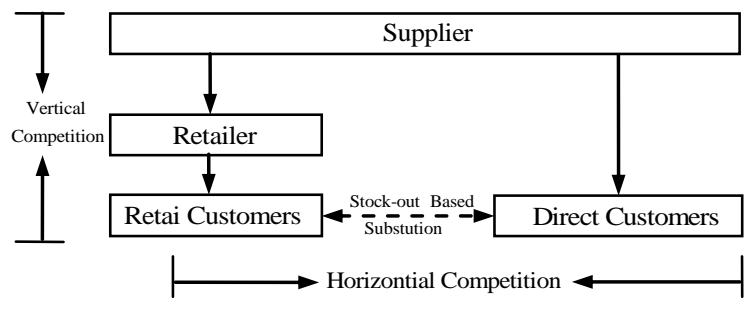

Figure 1. The Competition in a Dual-channel Supply Chain

\section{Model Description}

\subsection{Model Assumption}

The paper focus on the supply chain model with a single product in electronic and retail channel, only a supplier and a retailer in system, the supplier sets up the electronic channel, and the retailer manages the retail channel, the market consists of two kinds of customers: one is prefers the retail channel (is called as retail customers), the other is prefers the direct channel (is called as direct customers).

The product demand process $\{N(t), t \geq 0\}$ follows Poisson process with parameter $\lambda$, the proportion of direct customers is $\alpha(0 \leq \alpha \leq 1)$.

The supplier has no fixed ordering cost, and implements one-for-one ordering policy to replenish inventory, when a product demand reaches, a replenishment order will be send out immediately, the information lead time of replenishment is zero.

The retailer has a certain fixed ordering cost, and implements $(Q, R)$ ordering policy to replenish inventory, once inventory level reaches reorder point $R$, a replenishment order will be send out by supplier immediately, the economic ordering quantity is $Q$, and the information lead time of replenishment is zero.

The base-stock level of supplier is $S$.

When the retailer's replenishment arrives, if the supplier's inventory level cannot meet the retailer's economic ordering quantity $\mathrm{Q}$, supplier will stock up priority to retailer, and on account of ordering costs; the supplier will deliver goods in lot size Q.

There exist lost sales, once a stock-out occurs in the retailer, a proportional $\beta_{r}\left(0 \leq \beta_{r} \leq 1\right)$ of retailer customers will switch to electronic channel to buy products, otherwise, once a stock-out occurs in the supplier, a proportional $\beta_{d}\left(0 \leq \beta_{d} \leq 1\right)$ of direct customers will switch to retail channel to buy products. If a stock-out occurs in each distribution channel, customers who are unwilling to switch to the other channel to buy products will result in lost sales. In addition, when the supplier and the retailer are out of stock simultaneously, the customers will lose.

The replenishment lead time of supplier and retailer are independent exponential random variables with parameters $\mu_{s}$ and $\mu_{r}$, respectively. 
The reorder point of retailer is no-negative, that is to say $R \geq 0$, when a lot size $\mathrm{Q}$ arrive, the on-hand inventory level will always above the reorder point $\mathrm{R}$, so in a period of lead time, the retailer only has a lot size Q, this avoid the problem of crossed contract. In addition, this assumption guarantees the ordering goods in succession, and generates random lead time [10].

\subsection{Model Establishment}

We set up the profit model of supply chain by the Markov process.

According to assumption 1, we first obtain the following theorem.

Theorem 1 The arrival processes of direct and retail customers are independent, and follow the Poisson processes with parameter $\lambda_{d}=\alpha \lambda$ and $\lambda_{r}=(1-\alpha) \lambda$, respectively.

Proof: Let $D(t)$ and $R(t)$ denote the number of direct and retail customers in time interval $(0, t]$, respectively, then $N(t)=D(t)+R(t)$. Since the product demand process $\{N(t), t \geq 0\}$ is a Poisson process with parameter $\lambda$.

Let

$$
X(n)=\left\{\begin{array}{l}
1, \text { the } \mathrm{n} \text {-th arrival customer is a dierct customer } \\
0, \text { the } \mathrm{n} \text {-th arrival customer is not a dierct customer }
\end{array} \quad n=1,2,3, \ldots \ldots .\right.
$$

Then $P(X(n)=1)=\alpha, \quad P(X(n)=0)=1-\alpha, \quad E(X(n))=\alpha$, and $D(t)=\sum_{n=1}^{N(t)} X(n)$, so $D(t)$ is a compound Poisson process, and since $E(D(t))=E(N(t)) \cdot E(X(n))=\lambda \alpha t$, then $D(t)$ follows Poisson process with parameter $\lambda_{d}=\alpha \lambda$.

The same goes for that $R(t)$ follows Poisson process with parameter $\lambda_{r}=(1-\alpha) \lambda$.

$$
\begin{aligned}
P(D(t)=m, R(t)=n)= & \sum_{k=0}^{\infty} P(D(t)=m, R(t)=n \mid N(t)=k) P(N(t)=k) \\
& =P(D(t)=m, R(t)=n \mid N(t)=n+m) P(N(t)=n+m) \\
& =C_{n+m}^{m} \alpha^{m}(1-\alpha)^{n} \cdot \frac{e^{-\lambda t}(\lambda t)^{m+n}}{(n+m) !} \\
& =\frac{(n+m) !}{n ! m !} \alpha^{m}(1-\alpha)^{n} \cdot \frac{e^{-\lambda t}(\lambda t)^{m+n}}{(n+m) !} \\
& =\frac{(\alpha \lambda t)^{m}}{m !} e^{-\alpha \lambda t} \cdot \frac{((1-\alpha) \lambda t)^{n}}{n !} e^{-(1-\alpha) \lambda t} \\
& =P(D(t)=m) P(R(t)=n)
\end{aligned}
$$

So $D(t)$ and $R(t)$ are independent.

Let $x$ and $y$ denote the on hand inventory level of supplier and retailer, respectively, $(x, y)$ denotes the state of system, $\Omega$ is state space, then $(x, y) \in \Omega, \Omega=\{(x, y) \in Z \times Z \mid-Q \leq x \leq S, 0 \leq$ $y \leq R+Q$ \}. By assumption, we know that $y+Q>R$, but $y \geq 0$, so we have $R<Q$.

There are a total number of $(S+Q+1)(Q+R+1)$ states for the system.

To the current state $(x, y)$, there are five possible transitions characterized below:

Event $\langle 1>:(x, y) \rightarrow(x-1, y)$, a customer served from stock on hand by the supplier;

Event $<2>:(x, y) \rightarrow(x, y-1)$, a customer served from stock on hand by the retailer; 
Event $\langle 3\rangle:(x, y) \rightarrow(x-Q, y-1)$, a customer served from stock on hand by the retailer, and the retailer sends out a replenishment order;

Event $\langle 4>:(x, y) \rightarrow(x+1, y)$, a replenishment order arrives at the supplier;

Event $\langle 5\rangle:(x, y) \rightarrow(x, y+Q)$, a replenishment order arrives at the retailer

Figure 2 is the transition diagram of Markov model.

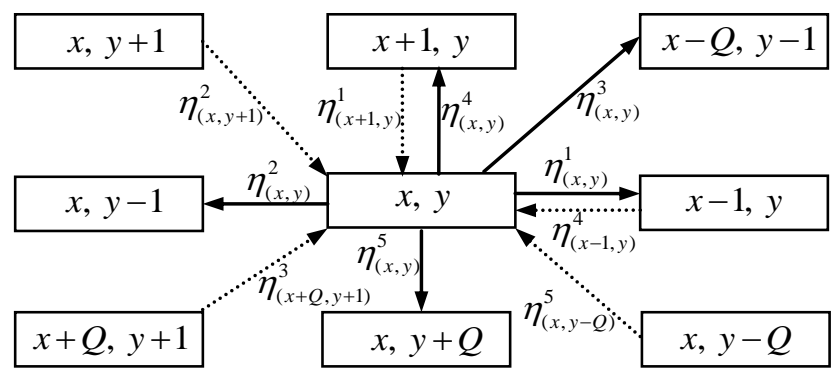

Figure 2. The Transition Diagram of Markov Model

In Figure 2, $\eta_{(x, y)}^{i}$ is the transition rate of event $i$ from state $(x, y)$, where $i=1,2,3,4,5$. Let $P_{(x, y)}^{(S, Q, R)}$ denotes the steady-state probability of state $(x, y)$. Obviously, if $(x, y) \notin \Omega$, then $P_{(x, y)}^{(S, Q, R)}=0$. Then we have the following state equilibrium equation:

$$
\begin{aligned}
& \sum_{i=1}^{5} \eta_{(x, y)}^{i} P_{(x, y)}^{(S, Q, R)}=\eta_{(x+1, y)}^{1} P_{(x+1, y)}^{(S, Q, R)}+\eta_{(x, y+1)}^{2} P_{(x, y+1)}^{(S, Q, R)}+\eta_{(x+Q, y+1)}^{3} P_{(x+Q, y+1)}^{(S, Q, R)}+\eta_{(x-1, y)}^{4} P_{(x-1, y)}^{(S, Q, R)}+\eta_{(x, y-Q)}^{5} P_{(x, y-Q)}^{(Q, R, S),} \\
& \text { Let } \phi_{(z)}=\left\{\begin{array}{ll}
1, & z>0 \\
0, & \mathrm{z} \leq 0
\end{array}, \quad \varphi_{(z)}=\left\{\begin{array}{ll}
1, & z \geq 0 \\
0, & z<0
\end{array}, \quad w_{(z)}=\left\{\begin{array}{ll}
1, & z=R+1 \\
0, & z \neq R+1
\end{array}, \quad \tau_{(z)}= \begin{cases}1, & z \leq R \\
0, & z>R\end{cases} \right.\right.\right.
\end{aligned}
$$

Then we can obtain the following transition rates:

$$
\begin{aligned}
\eta_{(x, y)}^{1}= & \phi_{(x)}\left[\lambda_{d}+\left(1-\phi_{(y)}\right) \beta_{r} \lambda_{r}\right] \\
\eta_{(x, y)}^{2}= & \phi_{(y)}\left(1-w_{(y)}\right)\left[\lambda_{r}+\left(1-\phi_{(x)}\right) \beta_{d} \lambda_{d}\right] \\
\eta_{(x, y)}^{3}= & \varphi_{(x)} w_{(y)}\left[\lambda_{r}+\left(1-\phi_{(x)}\right) \beta_{d} \lambda_{d}\right] \\
& \eta_{(x, y)}^{4}=(S-x) \mu_{S} \\
& \eta_{(x, y)}^{5}=\varphi_{(x)} \tau_{(y)} \mu_{r}
\end{aligned}
$$

Substituting (2)-(6) into (1), and sorting them, we can get the following linear equations system:

Constraint conditions:

$$
A^{(S, Q, R)} P^{(S, Q, R)}=0
$$

$$
\sum_{(x, y) \in \Omega} P_{(x, y)}^{(S, Q, R)}=1
$$

Where $A^{(S, Q, R)}$ is the transition rate matrix, $P^{(S, Q, R)}$ is the vector of steady-state probabilities, given $(S, Q, R)$ at will, we can work out the vector of steady-state probabilities $P^{(S, Q, R)}$ according to (1)-(8).

For example, given $(S, Q, R)=(2,1,0)$, the corresponding $A^{(2,1,0)} P^{(2,1,0)}=0$ is as equation (9).

\subsection{The Steady-state Sales Volume and Inventory Volume}


Suppose that the system operates over an infinite horizon. The supplier sells the product to the retailer at a per unit wholesale price $w$ and to customers at a fixed marginal price $d$ through its own direct channel. The costs incurred by the supplier for each unit of the product

$$
\left(\begin{array}{cccccccc}
3 \mu_{s} & 0 & 0 & -\lambda_{r}-\beta_{d} \lambda_{d} & 0 & 0 & 0 & 0 \\
0 & 3 \mu_{s} & 0 & 0 & 0 & 0 & 0 & 0 \\
-3 \mu_{s} & 0 & \mu_{r}+2 \mu_{s} & 0 & -\lambda_{d}-\beta_{r} \lambda_{r} & -\lambda_{r} & 0 & 0 \\
0 & -3 \mu_{s} & -\mu_{r} & 2 \mu_{s}+\lambda_{r}+\beta_{d} \lambda_{d} & 0 & -\lambda_{d} & 0 & 0 \\
0 & 0 & -2 \mu_{s} & 0 & \mu_{s}+\mu_{r}+\lambda_{d}+\beta_{r} \lambda_{r} & 0 & -\lambda_{d}-\beta_{r} \lambda_{r} & -\lambda_{r} \\
0 & 0 & 0 & -2 \mu_{s} & -\mu_{r} & \mu_{s}+\lambda_{d}+\lambda_{r} & 0 & -\lambda_{d} \\
0 & 0 & 0 & 0 & -\mu_{s} & 0 & \mu_{r}+\lambda_{d}+\beta_{r} \lambda_{r} & 0 \\
0 & 0 & 0 & 0 & 0 & -\mu_{s} & -\mu_{r} & \lambda_{d}+\lambda_{r}
\end{array}\right)\left(\begin{array}{c}
P_{(-1,0)} \\
P_{(-1,1)} \\
P_{(0,0)} \\
P_{(0,1)} \\
P_{(1,0)} \\
P_{(1,1)} \\
P_{(2,0)} \\
P_{(2,1)}
\end{array}\right)=\left(\begin{array}{l}
0 \\
0 \\
0 \\
0 \\
0 \\
0 \\
0 \\
0
\end{array}\right)
$$

sold through the retail channel and the direct channel are $c_{r}$ and $c_{d}$, respectively. In addition, the retailer's fixed ordering cost is $C$, the extra added ordering cost for unit product is $K$, the supplier and retailer incur a per unit inventory holding cost at rate $h_{s}$ and $h_{r}$, respectively. The retailer sells the product to customers at a fixed marginal retail price $r$. To avoid trivial problems, Let $m_{d}$ and $m_{r}$ be the respective margins of direct and retail sales, then $c_{d} \leq d$, $c_{r} \leq w \leq r, m_{d}=d-c_{d}, m_{r}=r-c_{r}$.

Then the steady-state expected sales volumes of supplier and retailer are as follows:

$$
\begin{gathered}
Q_{d}^{(S, Q, R)}=\lambda_{d} \sum_{x=1}^{S} \sum_{y=0}^{Q+R} P_{(x, y)}^{(S, Q, R)}+\beta_{r} \lambda_{r} \sum_{x=1}^{S} P_{(x, 0)}^{(S, Q, R)} \\
Q_{r}^{(S, Q, R)}=\lambda_{r} \sum_{x=-Q}^{S} \sum_{y=1}^{Q+R} P_{(x, y)}^{(S, Q, R)}+\beta_{d} \lambda_{d} \sum_{x=-Q}^{0} \sum_{y=1}^{Q+R} P_{(x, y)}^{(S, Q, R)}
\end{gathered}
$$

The steady-state expected inventory volumes of supplier and retailer are:

$$
\begin{aligned}
& I_{s}^{(S, Q, R)}=\sum_{x=1}^{S} \sum_{y=0}^{Q+R} x P_{(x, y)}^{(S, Q, R)} \\
& I_{r}^{(S, Q, R)}=\sum_{x=-Q}^{S} \sum_{y=1}^{Q+R} y P_{(x, y)}^{(S, Q, R)}
\end{aligned}
$$

The steady-state expected wholesale volume of retailer is:

$$
D^{(S, Q, R)}=Q \sum_{x=-Q}^{S} P_{(x, R)}^{(S, Q, R)}
$$

\section{The Profit of Supply Chain}

\subsection{The Centralized Supply Chain}

Suppose that all the prices are competitively determined, and all the cost-related parameters are exogenous, the decision variables in the system are $S, Q, R$, so the steady-state expected profit for the whole supply chain can be modeled as:

$$
\pi(S, Q, R)=m_{d} Q_{d}^{(S, Q, R)}+m_{r} Q_{r}^{(S, Q, R)}-h_{s} I_{s}^{(S, Q, R)}-h_{r} I_{r}^{(S, Q, R)}-\left(C+K D^{(S, Q, R)}\right)
$$

In the centralized supply chain, the goal is to find $S, Q, R$, and maximize the whole supply chain's profit.

We have the following theorem:

Theorem 2. There exists stock levels $\bar{S}, \bar{S}_{r}=\bar{Q}+\bar{R}$, such that $\pi(S, Q, R) \leq 0$ for all $S \geq \bar{S}$, $S_{r}=Q+R \geq \bar{S}_{r}$.

Proof : For some $\widehat{S}_{r}>0$, we have 


$$
\begin{aligned}
& \pi(S, \widehat{Q}, \widehat{R})=m_{d} Q_{d}^{(S, \hat{Q}, \hat{R})}+m_{r} Q_{r}^{(S, \hat{Q}, \widehat{R})}-h_{s} I_{s}^{(S, \hat{Q}, \widehat{R})}-h_{r} I_{r}^{(S, \hat{Q}, \widehat{R})}-\left(C+K D^{(S, \hat{Q}, \hat{R})}\right) \\
& \quad \leq m_{d}\left(\lambda_{d}+\beta_{r} \lambda_{r}\right)+m_{r}\left(\lambda_{r}+\beta_{d} \lambda_{d}\right)-h_{s} I_{s}^{(S, \hat{Q}, \widehat{R})}
\end{aligned}
$$

Therefore, in order to prove there exists a stock level $\bar{S}$, such that $\pi(S, \widehat{Q}, \widehat{R}) \leq 0$ for any $S>\bar{S}$, we only prove

$$
\begin{gathered}
\lim _{S \rightarrow \infty} h_{s} I_{s}^{(S, \hat{Q}, \widehat{R})}=\infty . \\
\text { Let } T_{S}=\sum_{y=0}^{\hat{Q}+\hat{R}} P_{S y}^{(S, \hat{Q}, \hat{R})} \text {, Then } h_{s} I_{s}^{(S, \hat{Q}, \widehat{R})}=h_{s} \sum_{x=1}^{S} \sum_{y=0}^{\hat{Q}+\hat{R}} x P_{(x, y)}^{(S, \hat{Q}, \widehat{R})} \geq h_{s} S \sum_{y=0}^{\hat{Q}+\hat{R}} P_{(S, y)}^{(S, \hat{Q}, \hat{R})}=h_{s} S T_{S} .
\end{gathered}
$$

Since $\liminf _{S \rightarrow \infty}\left\{T_{S}: S>0\right\}>0$, there exists natural number $N_{1}$ such that $l=\inf \left\{T_{S}: S>N_{1}\right\}>0$.

Let $L>0$, since $\lim _{S \rightarrow \infty} h_{s} S=\infty$, so exists natural number $N_{2}$ such that $h_{s} S>\frac{L}{l}$ for any $S>N_{2}$.

Let $N=\max \left\{N_{1}, N_{2}\right\}$, then when $S>N$, we have $h_{s} S T_{S}>l \frac{L}{l}=L$, so $\lim _{S \rightarrow \infty} h_{s} I_{s}^{(S, \bar{Q}, \bar{R})}=\infty$.

Similarly, we can prove that for some $\widehat{S} \geq 0$, there exists stock level $\bar{S}_{r}=\bar{Q}+\bar{R}$ such that $\pi(\widehat{S}, Q, R) \leq 0$ for all $S_{r}>\bar{S}_{r}$.

Sum up the above mentioned, theorem 2 holds.

Because of the complexity of profit function, we cannot derive the analytical solutions for $S, Q, R$, however, by theorem 2 we know that there exists sufficiently large stock level $S, Q, R$, such that $\pi(\bar{S}, \bar{Q}, \bar{R}) \leq 0$. So within the bounds of $\bar{S}, \bar{Q}, \bar{R}$, we can find the optimal stock level by complete numerical calculation.

\subsection{The Decentralized Supply Chain}

In the decentralized supply chain, the supplier and the retailer are all independent decision makers, the goal is maximize their own profits, ignoring the collective impact on the supply chain as a whole, so the profits of supplier and retailer are

$$
\begin{gathered}
\pi_{S}(S, Q, R)=m_{d} Q_{d}^{(S, Q, R)}+\delta m_{r} Q_{r}^{(S, Q, R)}-h_{s} I_{s}^{(S, Q, R)} \\
\pi_{r}(S, Q, R)=(1-\delta) m_{r} Q_{r}^{(S, Q, R)}-h_{r} I_{r}^{(S, Q, R)}-\left(C+K D^{(S, Q, R)}\right)
\end{gathered}
$$

respectively. Where $\delta=\frac{w-c_{r}}{r-c_{r}}$ is the coefficient of price double marginalization.

\section{MATLAB simulation}

In order to study the profit of supply chain, we designed the MATLAB program, firstly we find the state transition rate matrix $A^{(S, Q, R)}$, and get the vector of steady-state probabilities $P^{(S, Q, R)}$, so can obtain the profit of centralized and decentralized supply chain. To facilitate the problem discussion, in the above mentioned model, we assume $R=0$, other $R<Q$ condition can be discussed similarly, the basic parametric values in the model is as follows:

$$
\lambda=15, \alpha=0, \mu_{s}=5, \mu_{r}=10, h_{s}=220, h_{r}=250, m_{r}=m_{d}=100, C=30, K=5, \delta=0.5,
$$
$\beta_{r}=0.5, \beta_{d}=0.5$.

The computed result can see Table 1 and Table 2 . 
Table 1. The Profit $\pi(S, Q, 0)$ of Centralized Supply Chain

\begin{tabular}{|c|c|c|c|c|c|}
\hline$S$ & 1 & 2 & 3 & 4 & 5 \\
\hline 0 & 193 & 244 & 260 & 255 & 235 \\
\hline 1 & 362 & 370 & 349 & 313 & 265 \\
\hline 2 & 462 & 450 & 389 & 320 & 247 \\
\hline 3 & 474 & 467 & 391 & 293 & 196 \\
\hline 4 & 393 & 409 & 342 & 236 & 119 \\
\hline 5 & 241 & 281 & 232 & 140 & 20 \\
\hline 6 & 48 & 104 & 71 & -6 & -110 \\
\hline
\end{tabular}

Table 1 is the profit $\pi(S, Q, 0)$ of centralized supply chain when $S$ and $Q$ take different values, respectively, it can be seen from Table 1 that when $(S, Q, 0)$ take values $(3,1,0)$, the supply chain earns the maximum profit, the profit is 474 . So $(3,1,0)$ is the optimal inventory level of centralized supply chain.

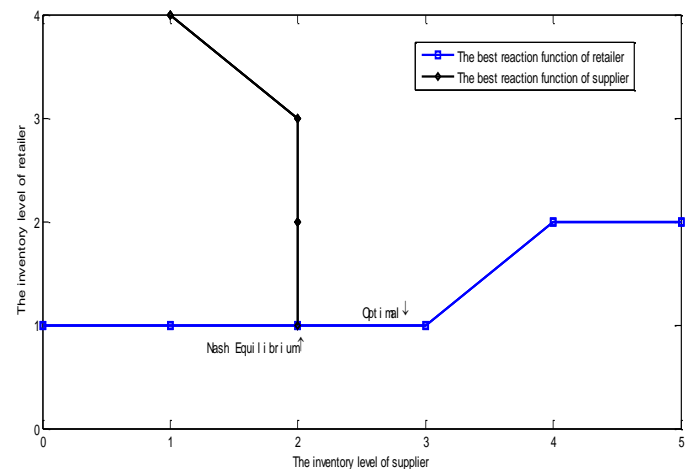

Figure 3. The Best Reaction Functions of Supplier and Retailer

Table 2 is the profit $\left(\pi_{S}, \pi_{r}\right)$ of decentralized supply chain when $S$ and $Q$ take different values, respectively, according to the best reaction functions of supplier and retailer (see Figure 3), we can find out the Nash Equilibrium point is $(2,1,0)$, the corresponding profits of supply chain are 318 and 145, respectively. See Table 2.

Table 2. The Profit $\left(\pi_{s}, \pi_{r}\right)$ of Decentralized Supply Chain

\begin{tabular}{|c|c|c|c|c|c|}
\hline$S$ & 1 & 2 & 3 & 4 & 5 \\
\hline 0 & 136,57 & 188,56 & 225,35 & 255,0 & $281,-46$ \\
\hline 1 & 247,115 & 269,101 & 285,64 & 298,15 & $308,-43$ \\
\hline 2 & 318,145 & 311,139 & 300,89 & 293,27 & $288,-41$ \\
\hline 3 & 315,159 & 306,161 & 282,109 & 255,38 & $235,-39$ \\
\hline 4 & 228,165 & 236,173 & 221,121 & 190,46 & $157,-38$ \\
\hline 5 & 75,166 & 104,177 & 104,128 & 88,52 & $57,-37$ \\
\hline 6 & $119,-167$ & $-75,179$ & $59,-130$ & $-60,54$ & $-74,-36$ \\
\hline
\end{tabular}

It can be seen from the above MATLAB simulation that there exists base-stock levels to maximize the profit of supply chain for supplier and retailer. But the optimal profit of supply chain system in the centralized decision-making is greater than the sum of supplier and retailer's profit in Nash Equilibrium. In the double action of vertical and horizontal 
competition, the decentralized decision-making system is more likely leads to competition penalty, damages the supply chain system's whole profit, increases the stock-out behavior of supply chain; and lowers the whole supply chain's efficiency.

The objective of supplier and retailer in the supply chain system are all maximize their own profits, the centralized supply chain system is more prone to increase the system's efficiency, and maximize the whole profit. So adopts the centralized supply chain system is better than decentralized one, in order to realize the supply chain coordination, we should design the supply chain contract, both sides may sign the profit sharing contract, and obtains a Pareto improvement of supplier and retailer's profits.

\section{Competition Analysis}

\subsection{Effect of Double Marginalization}

In the dual-channel supply chain, there exists vertical competition between supplier and retailer, it is mainly relates to double marginalization of price, we first analysis the effect of double marginalization to the efficiency of supply chain.

In Table 3, the proportion of direct customers $\alpha$ take values from 0 to 1 with step value 0.25 , the coefficient $\delta$ of double marginalization take values from 0 to 1 with step value 0.2 , in order to measure the channel efficiency, we introduce the competition penalty function [11]:

$$
P N=\frac{\pi\left(S^{*}, Q^{*}, R^{*}\right)-\pi_{S}\left(S^{* N}, Q^{* N}, R^{* N}\right)-\pi_{r}\left(S^{* N}, Q^{* N}, R^{* N}\right)}{\pi\left(S^{*}, Q^{*}, R^{*}\right)} \times 100 \%
$$

Table 3. Impact of Double Marginalization on Supply Chain Efficiency

\begin{tabular}{|c|c|c|c|c|c|c|c|c|c|c|c|c|c|c|c|}
\hline \multirow{3}{*}{$\delta$} & \multicolumn{3}{|c|}{$\alpha=0$} & \multicolumn{3}{|c|}{$\alpha=0.25$} & \multicolumn{3}{|c|}{$\alpha=0.5$} & \multicolumn{3}{|c|}{$\alpha=0.75$} & \multicolumn{3}{|c|}{$\alpha=1.00$} \\
\hline & \multicolumn{2}{|c|}{ Equilibrium } & \multirow{2}{*}{$\begin{array}{l}\text { PN } \\
(\%)\end{array}$} & \multicolumn{2}{|c|}{ Equilibrium } & \multirow{2}{*}{$\begin{array}{l}\mathrm{PN} \\
(\%)\end{array}$} & \multicolumn{2}{|c|}{ Equilibrium } & \multirow{2}{*}{$\begin{array}{l}\mathrm{PN} \\
(\%)\end{array}$} & \multicolumn{2}{|c|}{ Equilibrium } & \multirow{2}{*}{$\begin{array}{l}\mathrm{PN} \\
(\%)\end{array}$} & \multicolumn{2}{|c|}{ Equilibrium } & \multirow{2}{*}{$\begin{array}{l}\mathrm{PN} \\
(\%)\end{array}$} \\
\hline & $S$ & $Q, R$ & & $S$ & $Q, R$ & & $S$ & $Q, R$ & & $S$ & $Q, R$ & & $S$ & $Q, R$ & \\
\hline 0.0 & - & - & - & 4 & 3 & 0.0 & 4 & 2,0 & 0.0 & 5 & 1,0 & 0.0 & 6 & 0,0 & 0.0 \\
\hline 0.2 & 4 & 3,0 & 0.0 & 4 & 3 & 0.0 & 4 & 2,0 & 0.0 & 5 & 1,0 & 0.0 & 6 & 0,0 & 0.0 \\
\hline 0.4 & 4 & 3,0 & 0.0 & 3 & 2,0 & 6.50 & 4 & 1,0 & 13.8 & 5 & 1,0 & 0.0 & 6 & 0,0 & 0.0 \\
\hline 0.6 & 4 & 2,0 & 7.30 & 3 & 2,0 & 6.64 & 3 & 1,0 & 15.6 & 4 & 0,0 & 13.1 & 6 & 0,0 & 0.0 \\
\hline 0.8 & 3 & 2,0 & 63.0 & 3 & 1,0 & 52.1 & 2 & 0,0 & 43.1 & 4 & 0,0 & 16.1 & 6 & 0,0 & 0.0 \\
\hline 1.0 & 3 & 1,0 & 70.7 & 1 & 0,0 & 62.5 & 2 & 0,0 & 44.1 & 4 & 0,0 & 18.1 & 6 & 0,0 & 0.0 \\
\hline Optimal & 4 & 3,0 & & 4 & 3,0 & & 4 & 2,0 & & 5 & 1,0 & & 6 & 0,0 & \\
\hline
\end{tabular}

Note : $1 . \beta_{r}=\beta_{d}=0 ; 2$. Unless otherwise noted, the base parametric values in Table 1 are used, similarly hereinafter; 3. Shaded area indicates that the competitive base-stock levels perform at the optimal level in equilibrium.

In (17), $\pi\left(S^{*}, Q^{*}, R^{*}\right)$ denotes the maxim profit of centralized supply chain, $\pi_{S}\left(S^{* N}, Q^{* N}, R^{* N}\right)$, $\pi_{r}\left(S^{* N}, Q^{* N}, R^{* N}\right)$ denote the profit of supplier and retailer in equilibrium. From (18) we know that the more of competition penalty, the lower of channel efficiency. In Table 3, the competitive stock levels in equilibrium, the optimal stock levels and the corresponding competition penalty are reported for each $\alpha$ and $\delta$. We first make the following observation:

Result 1. With a low proportion of direct customers, increasing the degree of double marginalization intensifies understock behavior of the whole channel, increases the competition penalty, and thus decreases the efficiency of supply chain.

Result 2. For any given $\delta$, increase the rate of direct customers will result in the lower competition penalty, and thus increases the efficiency of supply chain. 


\subsection{The Combined Effect of Vertical and Horizontal Competition}

In the above 5.1 discussion, we assume that $\beta_{r}=\beta_{d}=0$, that is to say, we left out of the consideration of horizontal competition, next we discuss the combine effect of vertical and horizontal competition on channel efficiency.

Table 4. The Base-stock Level of Supply Chain

\begin{tabular}{ccccccccc}
\hline \multirow{2}{*}{$\beta_{r}=\beta_{d}$} & \multirow{2}{*}{ Optimal } & \multicolumn{6}{c}{ Nash equilibrium } \\
\cline { 2 - 8 } & & & \multicolumn{2}{c}{$\delta=0.25$} & \multicolumn{2}{c}{$\delta=0.5$} & \multicolumn{2}{c}{$\delta=0.75$} \\
\cline { 2 - 8 } & $S$ & $Q, R$ & $S$ & $Q, R$ & $S$ & $Q, R$ & $S$ & $Q, R$ \\
\hline 0.0 & $\mathbf{5}$ & $\mathbf{2 , 0}$ & 5 & 2,0 & 5 & 1,0 & 5 & 1,0 \\
0.2 & $\mathbf{5}$ & $\mathbf{2 , 0}$ & 5 & 2,0 & 5 & 1,0 & 5 & 1,0 \\
0.4 & $\mathbf{6}$ & $\mathbf{1 , 0}$ & 5 & 2,0 & 4 & 1,0 & 5 & 0,0 \\
0.6 & $\mathbf{6}$ & $\mathbf{1 , 0}$ & 5 & 2,0 & 6 & 1,0 & 5 & 0,0 \\
0.8 & $\mathbf{6}$ & $\mathbf{1 , 0}$ & 5 & 2,0 & 6 & 1,0 & 6 & 0,0 \\
1.0 & $\mathbf{6}$ & $\mathbf{0 , 0}$ & 5 & 1,0 & 6 & 0,0 & 6 & 0,0 \\
\hline
\end{tabular}

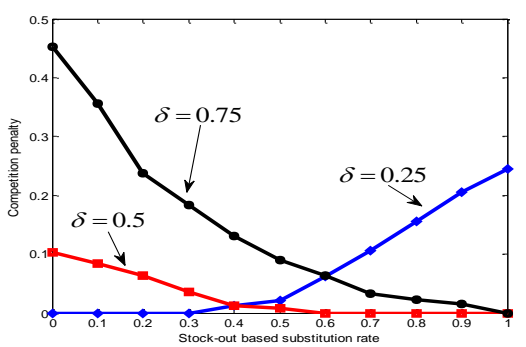

Figure. 4. Competition Penalty of Supply Chain

Firstly, we consider the symmetric condition of stock-out based substitution rate, that is $\beta_{r}=\beta_{d}$, we study that when the stock-out based substitution rate co-exists with double marginalization, the effect of stock-out based substitution rate on channel efficiency.

In Table 4 , the stock-out based substitution rate $\beta_{r}=\beta_{d}$ take values from 0 to 1 with step value 0.2 , the coefficient $\delta$ of double marginalization take values from 0.25 to 0.75 with step value 0.25 , we mainly consider 3 conditions of double marginalization (low: $\delta=0.25$; moderate: $\delta=0.5$; high: $\delta=0.75$ ), and examine the effect of increasing stock-out based substitution rate $\beta_{r}=\beta_{d}$ simultaneously, the outcome can see Table 4 and Figure 4 , we can get the following conclusion by analysis:

Result3. Increase the stock-out based substitution rates of direct and retail customers simultaneously, when the degree of double marginalization is high, diminishes the competition penalty, when the degree of double marginalization is low, intensifies the competition penalty. 
Table 5. Base-stock Level of Supply Chain

\begin{tabular}{|c|c|c|c|c|c|c|c|c|c|}
\hline \multirow{3}{*}{$\beta_{r}$} & \multirow{3}{*}{$\beta_{d}$} & \multirow{2}{*}{\multicolumn{2}{|c|}{$\begin{array}{c}\text { Optima } \\
\text { l }\end{array}$}} & \multicolumn{6}{|c|}{ Nash equilibrium } \\
\hline & & & & \multicolumn{2}{|c|}{$\delta=0.25$} & \multicolumn{2}{|c|}{$\delta=0.5$} & \multicolumn{2}{|c|}{$\delta=0.75$} \\
\hline & & $S$ & $Q, R$ & $S$ & $Q, R$ & $S$ & $\begin{array}{l}Q, \\
R\end{array}$ & $S$ & $Q, R$ \\
\hline 0.0 & 0.0 & 6 & 2,0 & 6 & 2,0 & & 2.0 & 5 & 1,0 \\
\hline 0.2 & 0.0 & 6 & 2, & 6 & 2,0 & 3 & 2,0 & 5 & 1,0 \\
\hline 0.4 & 0.0 & 6 & 1,0 & 6 & 2,0 & 0 & 0,0 & 5 & 1,0 \\
\hline 0.6 & 0.0 & 6 & 1,0 & 6 & 2,0 & 6 & 0,0 & 5 & 0,0 \\
\hline 0.8 & 0.0 & 5 & 1,0 & 6 & 1,0 & 5 & 1,0 & 5 & 0,0 \\
\hline 1.0 & 0.0 & 5 & $\mathbf{0 , 0}$ & 6 & 1,0 & 5 & 0,0 & 5 & 0,0 \\
\hline
\end{tabular}

Table 6. Base-stock Level of Supply Chain

\begin{tabular}{|c|c|c|c|c|c|c|c|c|c|}
\hline \multirow{3}{*}{$\beta_{d}$} & \multirow{3}{*}{$\beta_{r}$} & \multirow{2}{*}{\multicolumn{2}{|c|}{$\begin{array}{c}\text { Optima } \\
1\end{array}$}} & \multicolumn{6}{|c|}{ Nash equilibrium } \\
\hline & & & & \multicolumn{2}{|c|}{$\delta=0.25$} & \multicolumn{2}{|c|}{$\delta=0.5$} & \multicolumn{2}{|c|}{$\delta=0.75$} \\
\hline & & $S$ & $Q, R$ & $S$ & $Q, R$ & $S$ & $\begin{array}{l}Q, \\
R\end{array}$ & $S$ & $Q, R$ \\
\hline 0.0 & 0.0 & 6 & 2,0 & & 2,0 & & 1,0 & & 0,0 \\
\hline 0.2 & 0.0 & 6 & 2,0 & 6 & 2,0 & 5 & 2,0 & & 0,0 \\
\hline 0.4 & 00 & 6 & 2,0 & 6 & 1,0 & 5 & 2,0 & 5 & 1,0 \\
\hline 0.6 & 0.0 & 7 & 2,0 & 7 & 1,0 & 6 & 2,0 & 6 & 1,0 \\
\hline 0.8 & 0.0 & 7 & 3,0 & 6 & 3,0 & 6 & 3,0 & 6 & 2,0 \\
\hline 1.0 & 0.0 & 7 & 3,0 & 6 & 3,0 & 6 & 3,0 & 6 & 2,0 \\
\hline
\end{tabular}

Result 3 provides the combine effect of vertical and horizontal competition on supply chain under the condition $\beta_{r}=\beta_{d}$, in fact, when sock out occurs in some distribution channel, the stock out substitution rates are not identical in general case, so the asymmetric condition of stock out substitution rates deserve further study. Table 5, Table 6 and Figure 5 give the individual effect of $\beta_{r}$ and $\beta_{d}$ on the stock out behavior and efficiency of supply chain, respectively. We have the following conclusions by comparison:

Result 4. When the degree of double marginalization is low, increasing the substitution rate of supplier or retailer will intensify the competition penalty, and diminish the efficiency of supply chain. While the degree of double marginalization is moderate or high, increasing the substitution rate of supplier or retailer will diminish the competition penalty, and improve the efficiency of supply chain.

Given the above Table 4, Table 5 and Table 6, we have the following conclusion:

Result 5. In the centralized supply chain, the supplier and retailer can adjust the basedstock levels timely to different stock-out based substitution rate and benefit from it, but as independent decision makers in the decentralized supply chain, the supplier and retailer are more inattentive to customers' stock-out based substitution rate, therefore will result in greater competition penalty, and prone to lose their benefits.
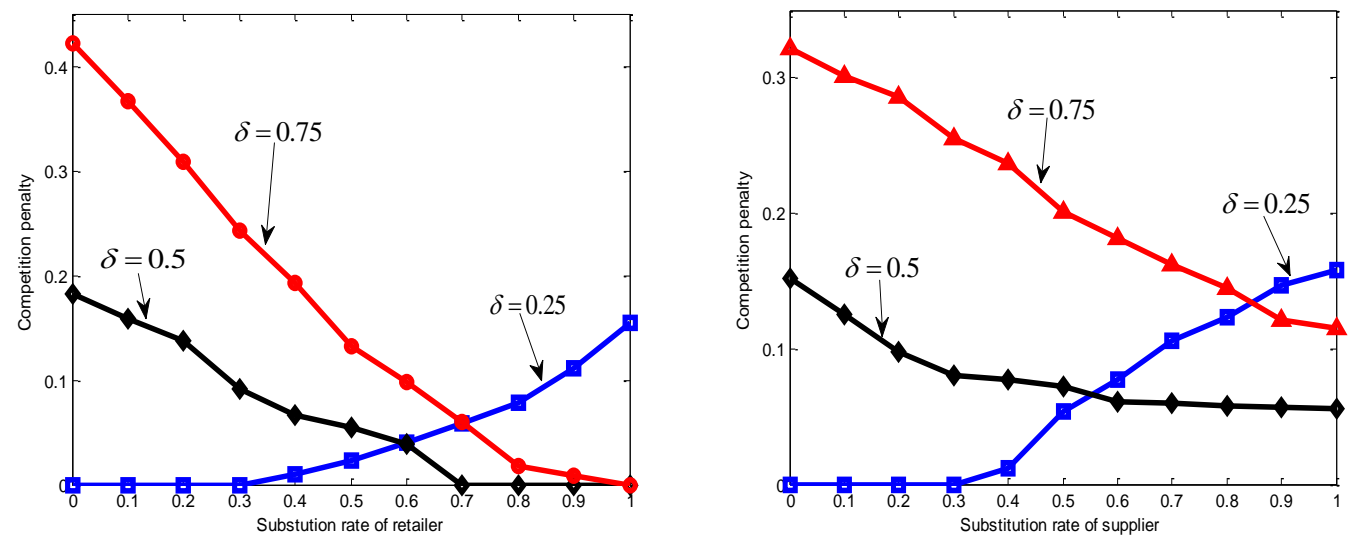

Figuer 5. The Competition Penalty of Supply Chain 


\section{Conclusions}

With the gradual maturity of e-commerce and the rapid development of third-party logistics, more and more enterprises have began to set up direct sales by introducing Internet channel. This paper studied the product availability in a dual-channel supply chain with $(Q, R)$ policy of retailer. According to the analysis of base stock level and efficiency of supply chain, we obtained some important insights: the centralized supply chain is sensitive to customers' stock-out based substitution rate, it can adjust the based-stock levels timely and benefit from stock-out based substitution rate, while the decentralized supply chain is more prone to influenced by stock-out based substitution rate, and leads to the inefficiency of supply chain.

In this paper, we assumed that the supplier adopt one for one ordering policy, if the supplier also adopts $(Q, R)$ policy, then the product availability problem of supply chain will be more complex, it deserves further discussion.

\section{Acknowledgements}

This work was supported by TianYuan Special Funds of the National Natural Science Foundation of China (No.11226337), Natural Science Foundation of Henan Province (No.122300410181, 132300410 373), Fund of Aeronautics Science (No.2013ZD55006), Natural Science Foundation of Henan education department (No.13A110399, 14A630019).

\section{References}

[1] J. J. Spengler, "Vertical integration and antitrust policy. Journal of Political Economy", vol. 58, no. 4, (1950).

[2] S. A. Lippman and K. F. McCardle, "The competitive newsboy, Operations Research.", vol. 45, no. 1, (1997).

[3] G. P. Cachon, "Stock wars: Inventory competition in a two echelon supply chain with multiple retailers", Operations Research, vol. 49, no. 5, (2001).

[4] S. Netessine and F. Zhang, "Positive Vs. Negative Externalities in Inventory Management: Implications for Supply Chain Design”, Manufacturing \& Service Operations Management, vol. 7, no. 1, (2005).

[5] R. Anupindi and Y. Bassok, "Centralization of stocks: Retailers vs. manufacturer", Management Science, vol. 45, no. 1 , (1999).

[6] G. Van Ryzin and S. Mahajan, "Supply chain coordination under horizontal competition", Working Paper, Graduate School of Business, Columbia University, New York, (2000).

[7] W. K. Chiang, D. Chhajed and J. D. Hess," Direct marketing, indirect revenues: A strategic analysis of dualchannel supply chain design", Management Science, vol. 49, no. 1, (2003).

[8] N. Kumar and R. Ruan, "On manufacturer's complementing the traditional retail channel with a direct online channel, Quantitative Marketing and Economics", vol. 4, no. 3, (2006).

[9] W.-y. Kevin Chiang, "Product availability in competitive an cooperative dual-channel distribution with stockout based substitution", European Journal of Operational Research, vol. 200, no. 1, (2010).

[10] Ma shihua and Lin Yong, “A inventory model based on stochastic lead time” , Computer Integrated Manufacturing Systems, vol. 8, no. 5, (2002).

[11] R. Caldentey and L. M. Wein, "Analysis of a decentralized production-inventory system", Manufacturing \& Service Operations Management, vol. 5, no. 1, (2003). 
International Journal of Hybrid Information Technology Vol. 7, No. 6 (2014) 